\title{
ON THE PROJECTIVE GREEN RING
}

\author{
SALAH EL DIN S. HUSSEIN AND ABD EL AZIZ A. RADWAN
}

\section{Introduction}

A natural strategy for studying the representation of a finite group $G$ is to consider a subgroup $H$ of $G$ whose representation is presumably simpler and try to use representations of $H$ to construct representations of $G$. The projective representations of finite groups arise naturally when one studies the relations between the representations of the group and representations of certain subgroups. The role played by the group rings in the linear representation theory is taken by the twisted group rings when one considers projective representations.

It was really J. A. Green who first introduced the notion of the representation ring $A(G)$ of a finite group $G$, in his paper [4], with the aim of studying the modular representations of $G$. The representation ring $A(G)$ is also called the Green ring. For a subgroup $H$ of $G$, the relation between the Green rings $A(G)$ and $A(H)$ has been established by D. J. Benson and R. A. Parker in [2], [3]. In an attempt to generalize the notion of the character of a representation of $G$ the concept of a species of the representation ring $A(G)$ has been introduced in [3].

Continuing this clue we define and investigate the projective Green ring $A^{T}(G)$ of a finite group $G$, where $T$ is any abelian group. Our definition is a generalization of the definition suggested by $H$. Opolka in [7]. After defining the projective Green $\operatorname{ring} A^{T}(G)$ we examine its general structure. We prove in Theorems (2.3) and (2.4) that if $H$ is any subgroup of $G$, then $A^{T}(G)$ is the direct sum of the kernel of the restriction map and the image of the induction map while $A^{T}(H)$ is the direct sum of the image of the restriction map and the kernel of the induction map. We round off this note by associating to each species $S$ of $A^{T}(G)$ an important conjugacy class of subgroups of $G$ called the origins of $S$. In order to define the origins of $S$ we need Theorem $(2.6)$ which states that $A^{T}(H)$ is integral over the image of the restriction map.

\section{Preliminaries}

In this section we recall some basic definitions and results on modules over twisted group algebras, full detail and proofs may be found in [5]. Throughout $G$ is a finite group

Received August 23, 1995.

1991 Mathematics Subject Classification. Primary 30C45.

Key words and phrases. 
with identity element $e, H$ is a subgroup of $G$, and $R$ is a commutative ring with identity. The group of units of $R$ will be denoted by $R$. All modules in this paper are left modules unless otherwise specified. Let $Z^{2}\left(G, R^{*}\right)$ be the group of 2-cocycles, $B^{2}(G, R)$ be the group of 2-coboundaries and $H^{2}(G, R)$ be the second cohomology group of $G$ over $R$. For every 2-cocycle $\alpha \in Z^{2}(G, R)$, the twisted group $\operatorname{ring} R^{\alpha} G$ is a free $R$-module with basis $\left\{\lambda_{g}: g \in G\right\}$ and multiplication defined by:

$$
a \lambda_{x} \cdot b \lambda_{y}=a b \alpha(x, y) \lambda x y, \text { for all } a, b \in R ; x, y \in G .
$$

If $\alpha: G \times G \rightarrow R$ is a 2-cocycle then the restriction of $\alpha$ to $H \times H$ is a 2-cocycle which will be also denoted by $\alpha$. If $V$ is an $R^{\alpha} G$-module, then we shall denote by $r_{G, H}(V)$, or $V_{H}$, the restriction of $V$ to $R^{\alpha} H$; thus as an $R$-module $V_{H}$ equals $V$ but only action of $R^{\alpha} H$ is defined on $V_{H}$. For every $R^{\alpha} H$-module $W$ we write $i_{H, G}(W)$, or $W^{G}$, for the induced $R^{\alpha} G$-module $\left(R^{\alpha} G \otimes_{R^{\alpha} H} W\right)$. We restrict our attention to finitely generated $R \alpha G$ modules which are $R$-free. If $V$ is an $R \alpha G$-module and $W$ is an $R^{\beta} G$-module then the module $V \otimes_{R} W$ is an $R^{\alpha \beta} G$-module where the action of the elements $\lambda_{g}, g \in G$, is defined by:

$$
\lambda_{g}(v \otimes w)=\lambda_{g} v \otimes \lambda_{g} w, \text { for all } v \in V, w \in W,
$$

and then extended to $V \otimes_{R} W$ and $R^{\alpha \beta} G$ by $R$-linearity,[5]. For every $g \in G$ and every $R^{\alpha} H$-module $V$ we define the module $V^{(g)}$ to be the $R^{\alpha}\left(g H g^{-1}\right)$-module whose underlying abelian group is $V$ and on which the elements $\lambda_{x}, x \in g H^{-1}$, act according to the rule:

$$
\lambda_{x} * v=\lambda_{g}^{-1} \lambda_{x} \lambda_{g} v, v \in V,[5] .
$$

For every $g \in G$, we shall write $H^{g}$ to denote the subgroup $g H g^{-1}$ of $G$.

Proposition 1.1. Suppose $H$ and $K$ are subgroup of $G, V$ is an $R^{\alpha} G$-module, and $W$ is an $R^{\alpha} H$-module. If $\omega$ is a set of double coset representatives for $(H, K)$ in $G$ then there are natural isomorphisms:

(i) $V \otimes W^{G} \cong\left(V_{H} \otimes W\right)^{G} \quad$ (as $R^{\alpha \beta} G$-modules)

(ii) $\left(W^{G}\right)_{K} \cong \oplus_{g \in \omega}\left[\left(w^{(g)}\right)_{H g \cap K}\right]^{K} \quad$ (as $R^{\alpha} K$-modules).

Proof. (i) Cf.[5], p. 218, Theorem (5.1).

(ii) Cf. [5], p. 227, Theorem (7.1).

\section{The Projective Green Ring.}

Throughout this section $R$ will be a field. For every $\alpha \in Z^{2}\left(G, R^{*}\right)$, all $R^{\alpha} G$-modules will be finitely generated $R^{\alpha} G$-modules. For simplicity we shall write $H \leq G$ to denote a subgroup $H$ of $G$ and $H<G$ to denote a proper subgroup $H$ of $G$.

Definition 2.1. Let $T$ be an abelian group with identity element $e^{\prime}$ and $\varphi: T \rightarrow$ $H^{2}(G, R)$ be a group homomorphism. For every $t \in T$ let $a^{t}(G)$ be the free abelian group 
generated by elements $\langle M\rangle$, one for each isomorphism class of $R^{\alpha} G$-modules, where $\alpha$ is any representative of the cohomology class $\varphi(t)$. If $Z$ and $C$ are, respectively, the integers and the complex numbers then we write $A^{t}(G)$ for the tensor product $C \otimes_{z} a^{t}(G)$. Let $A^{T}(G)=\oplus_{t \in T} A^{t}(G)$ and define addition and multiplication on the generators of $A^{T}(G)$ as follows:

$$
\langle M\rangle+\langle N\rangle=\langle M \oplus N\rangle,\langle M\rangle \cdot\langle N\rangle=\left\langle M \otimes_{R} N\right\rangle \text {. }
$$

If we extend those operations to $A^{T}(G)$ by C-linearity then clearly $A^{T}(G)$ becomes a commutative ring. We call the thing ring $A^{T}(G)$ the projective Green ring of $G$ over $R$.

Remarks 2.2. (1) It follows from the definition that $A^{T}(G)$ is a commutative Calgebra which is T-graded, i.e. $A^{t}(G) \cdot A^{s}(G) \subset A^{t s}(G)$ for every $t, s \in T$, [6]. Moreover, if $\alpha \in \varphi\left(e^{\prime}\right)$ and $R_{0}$ denotes the trivial $R^{\alpha} G$-module $R$ then $\left\langle R_{0}\right\rangle$ is the identity element of $A^{T}(G)$.

(2) The projective representation ring $A^{T}(H)$ of $H$ over $R$ is defined in a similar way with the understanding that for every $t \in T$ the cocycle $\alpha \in \varphi(t)$ is the restriction of $\alpha$ to $H \times H$. Clearly, the restriction map $r_{G, H}: A^{T}(G) \rightarrow A^{T}(H)$ is a ring homomorphism while the induction map $i_{H, G}: A^{T}(H) \rightarrow A^{T}(G)$ is a linear map which is not in general a ring homomorphism.

Theorem 2.3. For any subgroup $H$ of $G, A^{T}(G)$ is a direct sum of ideals:

$$
A^{T}(G)=\operatorname{Im}\left(i_{H, G}\right) \oplus \operatorname{Ker}\left(r_{G, H}\right) .
$$

Proof. The fact that $\operatorname{Im}\left(i_{H, G}\right)$ is an ideal is a direct consequence of Proposition (1.1) (i). We shall prove our assertion using mathematical induction on $|H|$. If $|H|=1$ then $R^{\alpha} H \cong R$ for every $\alpha \in Z^{2}(G, . R)$ It follows that $\operatorname{Im}\left(i_{H, G}\right)$ and $\operatorname{Im}\left(r_{G, H}\right)$ are freely generated by their identity elements. Hence,

$$
\begin{aligned}
\operatorname{CodimKer}\left(r_{G, H}\right) & =\operatorname{dim} A^{T}(G)-\operatorname{dimKer}\left(r_{G, H}\right) \\
& =\operatorname{dim} \operatorname{Im}\left(r_{G, H}\right) \\
& =\operatorname{dim} \operatorname{Im}\left(i_{H, G}\right) \\
& =1 .
\end{aligned}
$$

Since $\left.i_{H, G}(1)\right] \notin \operatorname{Ker}\left(r_{G, H}\right)$ then $\operatorname{Im}\left(i_{H, G}\right) \bigcap \operatorname{Ker}\left(r_{G, H}\right)=0$. Now assume that $|H|>1$ and for every proper subgroup $K$ of $H$,

$$
A^{T}(G)=\operatorname{Im}\left(i_{K, G}\right) \oplus \operatorname{Ker}\left(r_{G, K}\right)
$$

Using mathematical induction on the number of proper subgroups of $H$, one can easily obatin that

$$
A^{T}(G)=\sum_{k<H} \operatorname{Im}\left(i_{K, G}\right)+\bigcap_{K<H} \operatorname{Ker}\left(r_{G, K}\right) .
$$


Thus

$$
\operatorname{Im}\left(r_{G, H}\right)=r_{G, H}\left[\sum_{K<H} \operatorname{Im}\left(i_{K, G}\right)\right]+r_{G, H}\left[\bigcap_{K<H} \operatorname{Ker}\left(r_{G, K)}\right] .\right.
$$

Elementary calculations show that,

$$
r_{G, H}\left[\bigcap_{K<H} \operatorname{Ker}\left(r_{G, K}\right)\right]=\left[\bigcap_{K<H} \operatorname{Ker}\left(r_{H, K}\right)\right] \bigcap \operatorname{Im}\left(r_{G, H}\right) .
$$

Therefore,

$$
\operatorname{Im}\left(r_{G, H}\right)=r_{G, H}\left[\sum_{k<H} \operatorname{Im}\left(i_{K, G}\right)\right]+\left[\bigcap_{K<H} \operatorname{Ker}\left(r_{H, K}\right)\right] \bigcap \operatorname{Im}\left(r_{G, H}\right) .
$$

If follows that there are elements $b \in\left[\bigcap_{K<H} \operatorname{Ker}\left(r_{H, k}\right)\right] \cap \operatorname{Im}\left(r_{G, H}\right), a \in r_{G, H}\left[\sum_{K<H}\right.$ $\operatorname{Im}\left(i_{K, G}\right]$ such that $a+b=1$. If $N_{G}(H)$ is the normalize of $H$ in $G$ then $H^{g^{-1}} \cap H<H$ for every $g \notin N_{G}(H)$. Since $b \in \bigcap_{K<H} \operatorname{Ker}\left(r_{H, K}\right)$ then $b_{H^{g^{-1}}} \bigcap_{H}=0$ for every $g \notin N_{G}(H)$. If $\omega$ is a set of double coset representatives of $(H, H)$ in $G$ then, by Proposition (1.1), we have that

$$
\left(b^{G}\right) H \cong \oplus\left[\left(b^{(g)}\right)_{H^{g}} \bigcap_{H}\right]^{H} \cong \oplus\left[\left(b_{H^{-1}} \bigcap_{H}\right)^{(g)}\right]^{H},
$$

where the sum is taken over all $g \in \omega \bigcap N_{G}(H)$. Thus

$$
\left(b^{G}\right)_{H}=\left[N_{G}(H): H\right] b,
$$

showing that $\operatorname{Im}\left(r_{G, H}\right)=r_{G, H}\left(\operatorname{Im} i_{H, G}\right)$. It follows that for every $x \in A^{T}(G)$ there exists $y \in \operatorname{Im}\left(i_{H}, G\right)$ such that $x_{H}=y_{H}$. Therefore,

$$
x=y+(x-y) \in \operatorname{Im}\left(i_{H, G}\right)+\operatorname{Ker}\left(r_{G, H}\right),
$$

proving that $A^{T}(G)=\operatorname{Im}\left(i_{H, G}\right)+\operatorname{Ker}\left(r_{G, H}\right)$. Write $1 \in A^{T}(G)$ as $1=c^{G}+d$, where $c \in A^{T}(H), d \in \operatorname{Ker}\left(r_{G, H}\right)$. If $x \in \operatorname{Im}\left(i_{H, G}\right) \bigcap \operatorname{Ker}\left(r_{G, H}\right)$ then there exists $y \in A^{T}(H)$ such that $x=y^{G}$ and Proposition (1.1) entails that

$$
\left.x=x \cdot c^{G}+x \cdot d=\left(x_{H} \cdot c\right)\right)^{G}+\left(y \cdot d_{H}\right)^{G}=0
$$

This completes the proof of the theorem.

Theorem 2.4. For every subgroup $H$ of $G, A^{T}(H)$ is a direct sum of vector spaces:

$$
A^{T}(H)=\operatorname{Im}\left(r_{G, H}\right) \oplus \operatorname{Ker}\left(i_{H, G}\right) .
$$

Proof. For simplicity we shall write $E=\operatorname{Im}\left(r_{G, H}\right)+\operatorname{Ker}\left(i_{H, G}\right)$. Let $K$ be a subgroup of $H$. If $|K|=1$ and $\alpha \in Z^{2}\left(G, R^{*}\right)$ then for every $R^{\alpha} K$-module $M$ we have from Proposition (1.1) that,

$$
\left(M^{G}\right)_{H}=\oplus_{g \in \wedge}\left[\left(M^{(g)}\right)_{K}\right]^{H}=\oplus_{g \in \wedge} M^{H}
$$


. where $\wedge$ is a set of representatives of the left cosets of $H$ in $G$. Thus $\left(M^{G}\right)_{H}=[G$ : $H] M^{H}$. Hence $\left\langle M^{H}\right\rangle \in E$.

Suppose that, for every subgroup $K_{1}$ of $H$ such that $\left|K_{1}\right|<|K|$, if $\alpha \in Z^{2}\left(G, R^{*}\right)$ and $M$ be any $R^{\alpha} K_{1}$-module then $\left\langle M^{H}\right\rangle \in E$.

Now let $\alpha \in Z^{2}(G<R)$ and $M$ be any $R^{\alpha} K$-module. We have from Proposition (1.1) that

$$
\left(M^{G}\right)_{H} \cong \oplus_{g \in \omega}\left[\left(M^{(g)}\right)_{K^{g}} \bigcap_{H}\right]^{H},
$$

where $\omega$ is a set of double coset representatives of $(H, K)$ in G. Let $\omega_{1}=\left\{g \in \omega: K^{g} \mathbb{Z}\right.$ $H\}$ and $\omega_{2}=\left\{g \in \omega: K^{g} \leq H\right\}$

For every $g \in \omega_{2}$ we have that,

$$
\begin{aligned}
{\left[\left(M^{(g)}\right)_{K^{g}} \bigcap_{H}\right]^{H} } & =\left[\left(M^{(g)}\right)_{K^{g}}\right]^{H}=\left(M^{(g)}\right)^{H} \\
& =\left(M^{(g)}\right)^{H}-M^{H}+M^{H}
\end{aligned}
$$

If $g \in \omega_{1}$ then $\left|K^{g} \cap H\right|<|K|$ and it follows from the induction hypothesis that $\left\langle\left[\left(m^{(g)}\right)_{K^{g} \cap H}\right]^{H}\right\rangle \in E$.

Since $\left.\left\langle M^{(g)}\right)^{H}-M^{H}\right\rangle \in \operatorname{Ker}\left(i_{H, G}\right)$ and $\left\langle\left(M^{G}\right)_{H}\right\rangle \in \operatorname{Im}\left(r_{G, H}\right)$ then there exists a positive integer $p$ such that $p\left\langle M^{H}\right\rangle \in E$. Hence $\left\langle M^{H}\right\rangle \in E$ for every $R^{\alpha} K$-module $M$. Therefore $E=A^{T}(H)$.

Finally, if $y \in \operatorname{Im}\left(r_{G, H}\right) \bigcap \operatorname{Ker}\left(i_{H, G}\right)$ then there exists $u \in A^{T}(G)$ such that $y=u_{H}$. By Theorem (2.3) we can write the unite element 1 of $A^{T}(G)$ as $1=e_{1}+e_{2}$, where $e_{1}$ and $e_{2}$ are two orthogonal idempotent generators of $\operatorname{Im}\left(i_{H, G}\right)$ and $\operatorname{Ker}\left(r_{G, H}\right)$, respectively. Thus $e_{1}=x^{G}$ for some $x \in A^{T}(H)$. Moreover, using the first part of the proof we may write $x$ as $x=x_{1}+x_{2}$, where $x_{1}=v_{H}$, for some $v \in A^{T}(G)$, and $x_{2} \in \operatorname{Ker}\left(i_{H, G}\right)$. Invoking Proposition (1.1) we obtain,

$$
\begin{aligned}
y & =u_{H}=\left(u \cdot e_{1}\right)_{H}=\left(u \cdot x^{G}\right)_{H}=\left(u_{H} \cdot x\right)^{G} \\
& =\left(y \cdot v_{H}\right)^{G}+\left(u_{H} \cdot x_{2}\right)^{G} \\
& =y^{G} \cdot v+u \cdot x_{2}^{G}=0 .
\end{aligned}
$$

Therefore

$$
A^{T}(H)=\operatorname{Im}\left(r_{G, H}\right) \oplus \operatorname{Ker}\left(i_{H, G}\right)
$$

Corollary 2.5. For every $\alpha \in Z^{2}\left(G, R^{*}\right)$ and every subgroup $H$ of $G$ we have:

(i) If $V_{1}, V_{2}$ are $R^{\alpha} H$-modules such that $\left(V_{1}^{G}\right)_{H} \cong\left(V_{2}^{G}\right)_{H}$ then

$$
V_{1}^{G} \cong V_{2}^{G}
$$

(ii) If $W_{1}, W_{2}$ are $R^{\alpha} G$-modules such that $\left(W_{1_{H}}\right)^{G} \cong\left(W_{2_{H}}\right)^{G}$ then

$$
W_{1_{H}} \cong W_{2_{H}} \text {. }
$$


Proof. (i) The proof is a direct consequence of Theorem (2.3) since $\left\langle V_{1}^{G}\right\rangle-\left\langle V_{2}^{G}\right\rangle \in$ $\operatorname{Im}\left(i_{H, G}\right) \bigcap \operatorname{Ker}\left(r_{G, H}\right)$

(ii) Since $\left\langle W_{1_{H}}\right\rangle-\left\langle W_{2_{H}}\right\rangle \in \operatorname{Im}\left(r_{G, H}\right) \bigcap \operatorname{Ker}\left(i_{H, G}\right)$, then by Theorem (2.4) we have that $W_{1_{H}} \cong W_{2_{H}}$.

Definition 2.6.([3]) Let $B$ be a subalgebra of $A^{T}(G)$. A species $s$ of $B$ is a non-zero algebra homomorphism $\mathrm{s}: B \rightarrow C$.

In order to define the origin of a species we need the following integrality theorem. For definitions and properties of integral extensions of rings we refer to [1].

Theorem 2.7. For every subgroup $H$ of $G, A^{T}(H)$ is integral over $\operatorname{Im}\left(r_{G, H}\right)$.

Proof. By Proposition (5.3) of [3] we have that the Green ring $A(H)$ is integral over the image of the restriction map $\bar{r}_{G, H}: A(G) \rightarrow A(H)$. Let $Q$ be the kernel of the homomorphism $\varphi: T \rightarrow H^{2}(G, R)$. It is well known that $R^{\alpha} G \cong R G$ for every $q \in Q$, $\alpha \in \varphi(q),[5]$. Thus for every $q \in Q$ we have that

$$
\operatorname{Im}\left(\bar{r}_{G, H}\right) \cong \bar{r}_{G, H}\left(A^{e^{\prime}}(G)\right), \quad A^{q}(H) \cong A(H) .
$$

Therefore $A^{Q}(H)=\oplus_{q \in Q} A^{q}(H)$ is integral over $A_{1}=r_{G, H}\left(A^{e^{\prime}}(G)\right),[1]$. According to Theorem (3.2), Ch.2., of [5], if $\alpha \in Z^{2}(G, R)$ then there exists a positive integer $P$ such that $\alpha^{p} \in B^{2}(G, R)$. Thus, if $M$ is any $R^{\alpha} H$-module and $\langle M\rangle^{(p)}$ is the product in $A^{T}(H)$ of $p$ copies of $\langle M\rangle$ then $\langle M\rangle^{(p)} \in A^{Q}(H)$. It follow that $\langle M\rangle^{(p)}$ is integral over $A_{1}$. Hence $\langle M\rangle^{(p)}$ is integral over $\operatorname{Im}\left(r_{G, H}\right)$. This shows that $\langle M\rangle$ is integral over $\operatorname{Im}\left(r_{G, H}\right)$.

Proposition 2.8. Let $H$ be a subgroup of $G$ and $s$ be a species of $A^{T}(G)$. The following conditions are equivalent:

(i) $\operatorname{Ker}\left(r_{G, H}\right) \subseteq \operatorname{Ker}(s)$,

(ii) $\operatorname{Im}\left(i_{H, G}\right) \nsubseteq \operatorname{Ker}(s)$,

(iii) There is a species $t$ of $A^{T}(H)$ such that $s(x)=t\left(x_{H}\right)$ for all $x \in A^{T}(G)$.

Proof. (i) $\Rightarrow$ (ii). This is an easy consequence of Theorem (2.3) and the fact that $s$ is a non-zero homomorphism.

(ii) $\Rightarrow$ (i). Suppose that $\operatorname{Ker}\left(r_{G, H}\right) \nsubseteq \operatorname{Ker}(s)$. Let $x \in \operatorname{Ker}\left(r_{G, H}\right)-\operatorname{Ker}(s)$ condition (ii) implies the existence of an element $y \in \operatorname{Im}\left(i_{H, G}\right)-\operatorname{Ker}(s)$. Since $s(x)$ and $s(y)$ are non-zero elements of $C$ then $s(x, y)=s(x) s(y) \neq 0$. Thus $(x \cdot y)$ is a non-zero element of $\operatorname{Ker}\left(r_{G, H}\right) \cap \operatorname{Im}\left(i_{H, G}\right)$. This contradicts Theorem (2.3).

(i) $\Rightarrow$ (iii). Write $K=\operatorname{Ker}\left(r_{G, H}\right)$. Since $K \subset \operatorname{Ker}(s)$, the species $s$ induces a nonzero homomorphism $\bar{s}: A^{T}(G) / K \rightarrow C$. Thus $s$ induces a non-zero homomorphism $\bar{t}: \operatorname{Im}\left(r_{G, H}\right) \rightarrow C$, given by $\bar{t}\left(x_{H}\right)=s(x)$ for every $x \in A^{T}(G)$. Since $A^{T}(H)$ is integral over $\operatorname{Im}\left(r_{G, H}\right)$ then, by Proposition (1.8.1) of [2], There exists a non-zero algebra homomorphism $t: A^{T}(H) \rightarrow C$ such that $t\left(x_{H}\right)=\bar{t}\left(x_{H}\right)=s(x)$ for every $x \in A^{T}(G)$. 
(iii) $\Rightarrow(i)$. Obvious

Definition 2.9. A species $s$ of $A^{T}(G)$ factors through $H$ if and only if the equivalent conditions of Proposition (2.8) are satisfied. An origin of $s$ is a subgroup of $G$ which is minimal among those through which $s$ factors.

Proposition 2.10. Let $s$ be a species of $A^{T}(G)$. Then the origins of $s$ from a single conjugacy class of subgroups.

Proof. Let $H$ and $L$ be two origins of $s$. Since $H=\bigcup_{g \in G} H \cap L^{g}$ then

$$
\operatorname{Im}\left(i_{H, G}\right) \cdot \operatorname{Im}\left(i_{L, G}\right) \leq \oplus_{g \in G} \operatorname{Im}\left(i_{H} \cap L^{g, G}\right) .
$$

Since $s$ is a C-valued homomorphism then $\operatorname{Ker}(s)$ is a prime ideal. Thus

$$
\operatorname{Ker}(s) \nsupseteq \operatorname{Im}\left(i_{H, G}\right) \cdot \operatorname{Im}\left(i_{L, G}\right) .
$$

If follows that $\operatorname{Im}\left(i_{H} \bigcap_{L^{g}, G}\right) \not K \operatorname{Ker}(s)$ for some $g \in G$. Hence by minimality of $H$ we have that $H=H \cap L^{g}=L^{g}$.

\section{References}

[1] M. F. Atiyah and I. G. Macdonald, Introduction to Commutative Algebra, Addison-Wesley, Reading, Mass., 1969.

[2] D. J. Benson, Modular Representation Theory: New Trends and Methods, Springer-Verlag, LNM 1081, Berlin-Heidelberg, 1984.

[3] D. J. Benson and R. A. Parker, "The Green Ring of a Finite Group," J. Algebra, 87 (1984), 290-331.

[4] J. A. Green, "The modular representation algebra of a finite group, Illinois. J. Math, 6 (1962), 607-619.

[5] G. Karpilovski, Projective representations of a finite groups, Marcel Dekker Inc., 1985.

[6] C. Nastasescu and F. Van Oystaeyen, Graded Ring Theory, Math. Library, Vol. 28, North Holland, Amsterdam, (1982).

[7] H. Opolka, Rationalit ätsfragen bei Projektiven Darstellungen endlicher Gruppen, Ph.D. Dissertation, Universit ät M ünster, 1967.

Department of Mathematics, Faculty of Science, Ain Shams University, Cairo-Egypt.

Department of Mathematics, Faculty of Science, Ain Shams University, Cairo-Egypt. 